\title{
A New Load Shedding Scheme with Consideration of Distributed Energy Resources' Active Power Ramping Capability
}

\author{
Qiteng Hong, Member, IEEE, Liang Ji, Member, IEEE, Steven Blair, Senior Member, IEEE, Dimitrios \\ Tzelepis, Member, IEEE, Mazaher Karimi, Member, IEEE, Vladimir Terzija, Fellow, IEEE, and Campbell Booth
}

\begin{abstract}
This paper presents a novel load shedding scheme with consideration of the active power ramping capability of Distributed Energy Resources (DERs) to address the challenges due to low inertia and diverse types of DERs in microgrids. In the paper, it is demonstrated that due to the small inertia in microgrids, even with sufficient reserve power, the frequency could rapidly drop to a low level and trigger the DERs' under frequency protection (thus the total system collapse), if the reserve active power is not ramped up at a sufficient rate. The proposed load shedding scheme addresses this challenge by considering not only the DERs' reserve, but also their speed in injecting active power to the system to determine the amount of load should be shed, so that critical frequency thresholds are not violated. The proposed load shedding scheme is tested using a realistic real time hardware-in-the-loop arrangement. The results show that the proposed scheme can correctly detect the cases when the DERs' responses are too slow and trigger the required load shedding actions, thus effectively containing the frequency above the critical threshold.
\end{abstract}

Index Terms-Distributed energy sources, frequency control, islanding, load shedding, microgrids.

\section{NOMENCLATURE}

$H_{S M}^{i} \quad$ Inertia constant of the $i^{t h}$ synchronous machine in the microgrid

$S_{S M}^{i} \quad$ Capacity of the $i^{t h}$ synchronous machine in the microgrid

$K_{\max }^{i} \quad$ Maximum active power ramp rate of $i^{\text {th }}$ DER

$K_{\max } \quad$ Total maximum active power ramp rate of all DERs

$\bar{K} \quad$ Average active power ramp rate of all DERs

$K_{T} \quad$ Total active power ramp rate of all DERs

$\bar{K}_{\text {min }} \quad$ Minimum required active power ramp rate to avoid violating the lowest frequency limit

$f_{n} \quad$ Nominal frequency of the system

$f_{0} \quad$ Measured frequency at the current moment

$f_{p u} \quad$ System frequency in per unit

$f_{L} \quad$ Lowest acceptable frequency limit

$f_{\text {safe }} \quad$ Low frequency safety threshold

$f_{E D} \quad$ Event detection frequency threshold

$\Delta f_{\max } \quad$ The maximum allowable frequency deviation from the current frequency point

RoCoF Rate of Change of Frequency

$\mathrm{RoCOF}_{0} \quad$ RoCoF measured at the current time instance

$R o C o F_{\max }$ Maximum RoCoF measured in a frequency event

RoCoF $F_{E D}$ Event detection RoCoF threshold

$T_{0}$
$T_{E D}$
$T_{c}$
$\Delta P_{E}$
$\Delta P_{0}$
$\Delta P(t)$
$P_{R}^{T}$
$P_{R}^{i}$
$P_{R}(t)$
$P_{\text {shed }}$
$P_{e}(t)$
$P_{m}(t)$
$R_{i}$

$\Delta P_{\text {ref }}^{i}$

Current time instance

Time when the event is detected

Time when the frequency will drop to $f_{L}$

Initial power imbalance

Current power imbalance

Dynamic power imbalance

Total reserve power

Active power reserve of $i^{t h}$ DER

Responding active power from DERs

Calculated amount of load to be shed

Total electrical power in the microgrid

Total mechanical power from generators

Droop setting of the $i^{t h}$ DER that provides frequency regulation

Power reference change of the $i^{\text {th }}$ DER with droop control

\section{INTRODUCTION}

$\mathbf{U}$ NDER frequency load shedding is considered as a critical measure for balancing power generation and demand in microgrids during emergency frequency events [1]. When a microgrid gets disconnected from the main grid due to unexpected events (e.g. faults) and the local generation is smaller than the demand, the microgrid may experience severe and rapid frequency degradation due to the relatively small system inertia level [2], [3]. Effective load shedding is therefore required to curtail a certain amount of load so that the power quality can be maintained at a satisfactory level and the supply to critical loads can be sustained [4]. Underfrequency load shedding has been investigated extensively in the research community, and a comprehensive review of the exiting load shedding methods for microgrids is presented in [1]. The existing methods can be broadly classified into three main categories: static frequency/Rate of Change of Frequency (RoCoF) load shedding [5]-[7], adaptive load shedding [3], [8]-[20] and load shedding using Artificial Intelligence (AI) techniques [21]-[30].

Static frequency load shedding is a conventional method that has been applied widely for both large power systems and microgrids [3], [5]. It operates based on pre-defined frequency thresholds and sheds a certain amount of load when the frequency falls below these thresholds for pre-defined periods of time. Such schemes are relatively easy to implement, but do not have awareness of the magnitude of power imbalance, 
and simply react to the measured frequency, which could result in over/under shedding of loads, leading to an exacerbation of the disturbance [11]. RoCoF-based load shedding evaluates the RoCoF values at a number of pre-defined frequency levels, and if the measured RoCoF magnitude is larger than the pre-set limits, a corresponding amount of load will be shed [6]. This method relies on accurate algorithms for frequency and RoCoF measurements [31]. In [6], a combination of RoCoF and fixed frequency-based load shedding is implemented. The work focuses on the scenarios of intentional islanding from grid connected mode when the required loads are shed before the islanding event occurs. In [7], the RoCoF-based load shedding is used along with the consideration of customers' willingness to pay for maintaining the supply to determine the priority ranking of different loads. RoCoF-based schemes provide a level of improved adaptability than the fixed frequency based schemes as the RoCoF value is proportional to the amount of power imbalance. However, the load shed in each step is still fixed, i.e. the adaptability to an event is limited. This is particularly the case for a microgrid environment, where the operating condition can change significantly with different amounts of reserve power and operating statuses of the Distributed Energy Resources (DERs) [32].

Adaptive load shedding schemes have gained significant interests in recent years [3], [8]-[20], [33]. The key advantage of adaptive schemes is the capability to adaptively take different actions based on varied system conditions. The provision of adaptability can be realized in different ways. For example, in [8], adaptive load shedding actions are deployed by considering the droop control characteristics of inverter-based DERs; in [9], an analytical approach is taken to adaptively adopt the required the load to be shed; in [10], wide-area real time measurement signals are used to dynamically assess the real time system stability margins and operational limits, thus to determine the amount of loads need to be shed once incidents occur. A key element of adaptive schemes is the capability to deal with different sizes of power imbalance. Therefore, load shedding actions based on the estimation of power imbalance is another widely used method for providing adaptive load shedding [3], [11], [12]. The fundamental principle of this type of adaptive scheme is based on the estimation of the power imbalance size, then comparing it with the available reserve in the system in order to determine the amount of load required to be shed. The estimation of power imbalance is achieved by firstly finding the overall equivalent inertia level of the microgrid and then calculating the power deficiency based on the measured RoCoF using the swing equation [34]. For example, this method is applied in [3] along with a state estimation technique to estimate the load flow in each branch so as to estimate the amount of power imbalance during sudden islanding and load increase scenarios. In [12], a multistage under frequency load shedding scheme was proposed, where the load planned to be shed at each stage is also based on the estimation of power imbalance.

While the aforementioned adaptive load shedding schemes are capable of adapting the shedding action to different system conditions, there are still a number of critical shortcomings that have not been addressed: 1) frequency control in mi- crogrids not only depends on the available reserve power when a frequency event occurs, but is also highly dependent on the speed with which the resources can respond to the event. According to the studies conducted in [35], the active power ramp rate of the DERs has a dominating impact on the frequency profile. When a sudden islanding event occurs, if the load shedding scheme only considers the available reserve without consideration of the DERs' active power ramping capability, it could lead to the frequency dropping to an unacceptable level, which could result in tripping of DERs due to under frequency protection, potentially leading to whole system collapse, even there is sufficient reserve energy available. 2) the existing methods based on power imbalance estimation assume that the DERs will deliver the power as required, without a proper mechanism to deal with the cases where DERs under deliver the levels of power required. From the GB power cut event in 2019, it is observed that, due to various reasons, DERs may deliver smaller amounts of power than required and expected [36].

AI techniques have also been used for load shedding purpose. In [21], [22], power system models are used to train neural networks so as to predict the system behavior, in order to determine the load to be shed under various scenarios. In [23], [24], genetic algorithms are used to find the optimal amount of load that needs to be shed. [25] presents an emergency load shedding scheme based on deep Q learning, where the load priority and the anticipated effect of shedding actions considering loads' frequency dependent characteristics are formulated in the reward function, thus to achieve fast frequency recovery, while maintaining the power supply to critical loads. The Q-learning algorithm is also applied in [26], where the load shedding amount is determined by considering the frequency droop characteristics of DERs, and the load priority is formulated as the reward for the Q-learning algorithm to minimize the interruption to critical loads. In [27], a load shedding approach that integrates the model-driven and datadriven methods for frequency prediction and load shedding amount calculation is proposed. [28] proposes the use of multiagent systems to coordinate the load shedding actions to eliminate the need for communications. These publications provide valuable insights in demonstrating the capability of AI-based methods to enable effective load shedding actions, but the DERs' active power ramping capability is not considered, which could lead to frequency dropping to an unacceptable level even with sufficient reserve. In [29], deep reinforcement learning is applied for adaptively determining the emergency control actions (including load shedding), but the key focus for shedding action is mainly placed on voltage stability without consideration of the frequency in the system. In [30], an effortbased reward approach is proposed and implemented as a multi-agent system for allocating the load shedding amount in networked microgrids, where an effort index that considers the microgrid's contribution to the network and its capacity is defined to determine the amount of load to be shed. The proposed system aims to provide a fair chance for small micrgrids in supporting the network operation. However, this apporach is mainly designed for scheduled load shedding actions and is not suitable for addressing emergency frequency 
deviation during power imbalance events.

In addition to aforementioned publications, there are some other activities reported in the area of load shedding for microgrid applications: [37] and [38] present methods that aim to consider both voltage and frequency stability when issuing load shedding actions; [39] specifically considers the survivability issues of synchronous DERs when determining loads to be shed; Similar to adaptive load shedding schemes, the aforementioned schemes do not consider the DERs' active power ramping capability (which is often widely diverse depending on the DER technology and interface), and this could lead to failure in recovering the system following severe events.

To address the aforementioned challenges associated with non- or inadequate consideration of DERs' active power ramping capability, this paper presents a novel load shedding scheme that dynamically calculates the required amount of load to be shed with consideration of the DERs' reserve power as well as their active power ramping capabilities, which ensure the minimum amount of load is shed while the critical frequency limit is not violated, thus avoiding the risk of frequency collapse. Compared with existing load shedding schemes, the propose method is not only adaptive to the magnitude of power imbalance, but also adaptive to the DERs' active power ramping capability in the post-disturbance and load shedding phases of the disturbance management process. Another key advantage of the proposed scheme is that by evaluating the required ramping rate from DERs, it is also capable of shedding an appropriate amount of load when DERs fail to deliver the required response.

The paper is organized as follows: Section II presents an overview of frequency control in microgrids; Section III presents the design of the proposed load shedding scheme; Section IV presents case studies where a highly realistic Hardware-in-the-Loop (HiL) test setup has been used to demonstrate the effectiveness of the proposed scheme. Section $\mathrm{V}$ presents the conclusions of this work.

\section{Frequency Control in Microgids}

\section{A. Frequency response from DERs in microgrid}

When power imbalance events occur, microgrids rely on dispatchable DERs to adjust their active power output to rebalance the system. Typically, droop control is used by DERs for regulating the frequency by sharing the active power injection contribution. For the droop control, if the measured frequency at the current time instance is $f_{0}$, then the output power reference of DERs will be adjusted based on how much $f_{0}$ deviates from $f_{n}$ and the droop setting $R_{i}$, which can be described using (1):

$$
\Delta P_{\text {ref }}^{i}=-\left(1 / R_{i}\right)\left(f_{0}-f_{n}\right)
$$

\section{B. Frequency behavior during power imbalance events}

The frequency behavior of a microgrid is governed by the swing equation as shown in (2) [34]: $\frac{2 \sum H_{S M}^{i} S_{S M}^{i}}{f_{n}} \times f_{p u} \times R o C o F(t)=P_{m}(t)-P_{e}(t)=\Delta P(t)$

Assuming the system operates at the nominal frequency (i.e. $f_{p u}=1 \mathrm{pu}$ ) before disturbance (e.g. sudden islanding), the RoCoF value of the system can be described using (3):

$$
\operatorname{RoCoF}(t)=\frac{\Delta P(t) \times f_{n}}{2 \sum H_{S M}^{i} S_{S M}^{i}}
$$

$\Delta P(t)$ is the amount of active power imbalance and it is a time-varying function of the initial power imbalance $\Delta P_{E}$ and the responding power $P_{R}(t)$ from DERs as shown in (4):

$$
\Delta P(t)=\Delta P_{E}+P_{R}(t)
$$

$\Delta P_{E}$ is the largest power imbalance during an event, which occurs at the beginning of the event. This is because following the event occurrence, the response from $P_{R}(t)$ will decrease the power imbalance. Therefore, $\left|\Delta P_{E}\right|$ is also referred to as the size of power imbalance event in the rest of the paper.

From (3) and (4), the RoCoF value can be described as:

$$
\operatorname{RoCoF}(t)=\frac{\left(\Delta P_{E}+P_{R}(t)\right) \times f_{n}}{2 \sum H_{S M}^{i} S_{S M}^{i}}
$$

The frequency deviation $\Delta f$ over time is:

$$
\Delta f=\int \operatorname{RoCoF}(t) d t=\int \frac{\left(\Delta P_{E}+P_{R}(t)\right) \times f_{n}}{2 \sum H_{S M}^{i} S_{S M}^{i}} d t
$$

Assuming the current time instance is $T_{0}$ with a power imbalance of $\Delta P_{0}$, the system is with a frequency $f_{0}$, the absolute lowest frequency the system can tolerate is $f_{L}$ (which is often the under frequency protection threshold for DERs [40]), the DERs have an overall active power ramping rate $K_{T}$ at $T_{0}$ and they will maintain this rate, then the maximum permitted frequency deviation $\Delta f_{\max }$ can be expressed as:

$$
\Delta f_{\text {max }}=f_{L}-f_{0}=\int_{T_{0}}^{T_{c}} \frac{\left(\Delta P_{0}+K_{T} \times t\right) \times f_{n}}{2 \sum H_{S M}^{i} S_{S M}^{i}} d t
$$

where $T_{c}$ is the time when the frequency will drop to $f_{L}$. (7) indicates that, if the DERs continue to ramp at a constant rate of $K_{T}$, the system will reach $f_{L}$ at $T_{c}$. As $T_{0}$ is the current time instance, for simplicity, $T_{0}$ is set to 0 . Therefore, (7) can be expressed as:

$$
\begin{aligned}
\Delta f_{\text {max }} & =\int_{0}^{T_{c}} \frac{\left(\Delta P_{0}+K_{T} \times t\right) \times f_{n}}{2 \sum H_{S M}^{i} S_{S M}^{i}} d t \\
& =\frac{\left(\Delta P_{0} \times T_{c}+\frac{K_{T}}{2} \times T_{c}^{2}\right) \times f_{n}}{2 \sum H_{S M}^{i} S_{S M}^{i}}
\end{aligned}
$$

Solving (8), the solutions are:

$$
T_{c}=\frac{-\Delta P_{0} \pm \sqrt{\Delta P_{0}^{2}+\frac{4\left(f_{L}-f_{0}\right) \times K_{T} \times \sum H_{S M}^{i} S_{S M}^{i}}{f_{n}}}}{K_{T}}
$$


(8) and (9) mean that at any given time $T_{0}$ during the frequency event with a measured frequency $f_{0}$ and a frequency response ramp rate of $K_{T}$, if real positive roots can be found, that means the frequency will eventually drop to the limit $f_{L}$, which could cause severe consequences, e.g. DERs tripping, and thus a total system collapse. In order to avoid the frequency dropping to $f_{L}, K_{T}$ should be large enough, i.e. the DERs' active power ramping rate should be fast enough. Referring to (9), if no real root for $T_{c}$ can be found, the system will not reach the lowest limit. In order to meet this requirement, the following condition must be met:

$$
\Delta P_{0}^{2}+\frac{4 \times\left(f_{L}-f_{0}\right) \times K_{T} \times \sum H_{S M}^{i} S_{S M}^{i}}{f_{n}}<0
$$

Solving (10), $K_{T}$ needs to meet the following condition:

$$
K_{T}>\underbrace{\frac{\Delta P_{0}^{2} \times f_{n}}{4 \times\left(f_{0}-f_{L}\right) \times \sum H_{S M}^{i} S_{S M}^{i}}}_{\bar{K}_{\text {min }}}
$$

It should be noted that $K_{T}$ represents the combined ramp rate of all DERs at $T_{0}$, while $\bar{K}_{\text {min }}$ is the required minimum average power ramp rate to avoid frequency dropping to $f_{L}$. Both $K_{T}$ and $\bar{K}_{\min }$ are time varying variables and can be calculated and evaluated every time step. Fig. 1 shows an example how $\bar{K}_{\text {min }}$ will change with different values of $f_{0}$ and the initial power imbalance $\Delta P_{E}$. It can be seen that, with the same initial power imbalance $\Delta P_{E}$, the value of $\bar{K}_{\text {min }}$ will increase as the frequency drops. For DERs, the response rate $K_{T}$ can also change over time, and generally it will tend to decrease as it approaches the DERs approaches to new settling points. The evaluation of the value for $K_{T}$ will be further discussed in Section III-E. Therefore, if $\bar{K}_{\text {min }}$ is greater than $K_{T}$, it means the DERs are not going to be sufficiently fast to avoid the frequency dropping below the lowest limit $f_{L}$ even there is enough reserve power available. This is because $\bar{K}_{\min }$ will keep increasing and the reserve power is injected to the system too slowly, so the system could collapse. Therefore, while the total reserve power in the system is a critical element to be considered for load shedding, the active power ramping capability is also key to ensure the system can survive during severe frequency events.

\section{DESIGN OF THE LOAD SHEDDING SCHEME}

\section{A. Overview of the load shedding scheme}

The proposed load shedding algorithm contains five main stages, which is illustrated in the flow chat in Fig. 2 and the diagram in Fig. 3. Stage I is to monitor the operational mode of the microgrid (i.e. grid-connected or islanded) and detect power imbalance events - this will determine whether to activate the load shedding scheme in the subsequent stages; if there is a frequency event detected in islanded mode, Stage II will calculate the amount of the power imbalance; Stage III caters for over-load conditions, i.e. if the total reserve is not sufficient to meet the calculated power imbalance, the system will shed load corresponding to the overload; Stage IV evaluates the DERs' active ramping capability and the

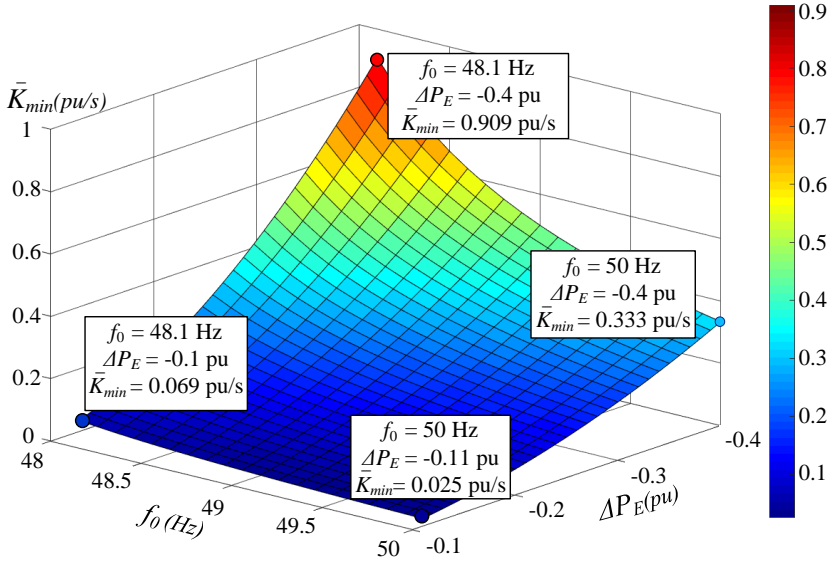

Fig. 1. The required active power ramp rate $\bar{K}_{\text {min }}$ at different $f_{0}$ and $\Delta P_{E}$

minimum required ramp rate to determine whether the DERs respond too slow to contain the frequency deviation to make load shedding decisions; Between the shedding actions, a holding time $T_{H o l d}$ is introduced to account for potential communication delay and the system's measurement delay to avoid consecutive commands before the last load shedding action takes effect. For the final stage, when the frequency drops to a level close to the critical limit (i.e. $f_{\text {safe }}$ ) and there is not enough time to further wait for the DERs to ramp up their response, so it will shed the remaining power imbalance in system to avoid dropping to the lowest permitted value $f_{L}$.

\section{B. Stage I: Detection of the microgrid's operational mode and frequency events}

Microgrids typically have two operational modes, i.e. grid connected and islanded modes, which have significantly different frequency control and load shedding strategies. The first stage of the algorithm is to monitor the system through continuous measurement of frequency and RoCoF signals to determine whether there is a credible under-frequency event, by comparing the measured frequency and $\mathrm{RoCoF}$ with preset thresholds $f_{E D}$ and $R o C o F_{E D}$. If both of the $f_{E D}$ and $R o C o F_{E D}$ are violated, the scheme will consider there is a frequency event is detected, and then an islanding detection algorithm will be executed to determine whether the system is under islanded mode. The proposed load shedding algorithm is designed for a microgrid control system as reported in [41]. As the islanding detection is not the main focus of the paper, the detailed implementation of the islanding detection for the system [41] can be found in [42]. The subsequent load shedding actions will only be triggered when an underfrequency event is detected the in the islanded mode.

\section{Stage II: Evaluation of the magnitude of power imbalance}

Once an under-frequency event in islanded mode is detected, the algorithm will move to Stage II to evaluate the power imbalance using (12), where $R o C o F_{0}$ is RoCoF measured at the current time step and it will vary over time.

$$
\Delta P_{0}=\frac{2 \sum H_{S M}^{i} S_{S M}^{i}}{f_{n}} \times R o C o F_{0}
$$




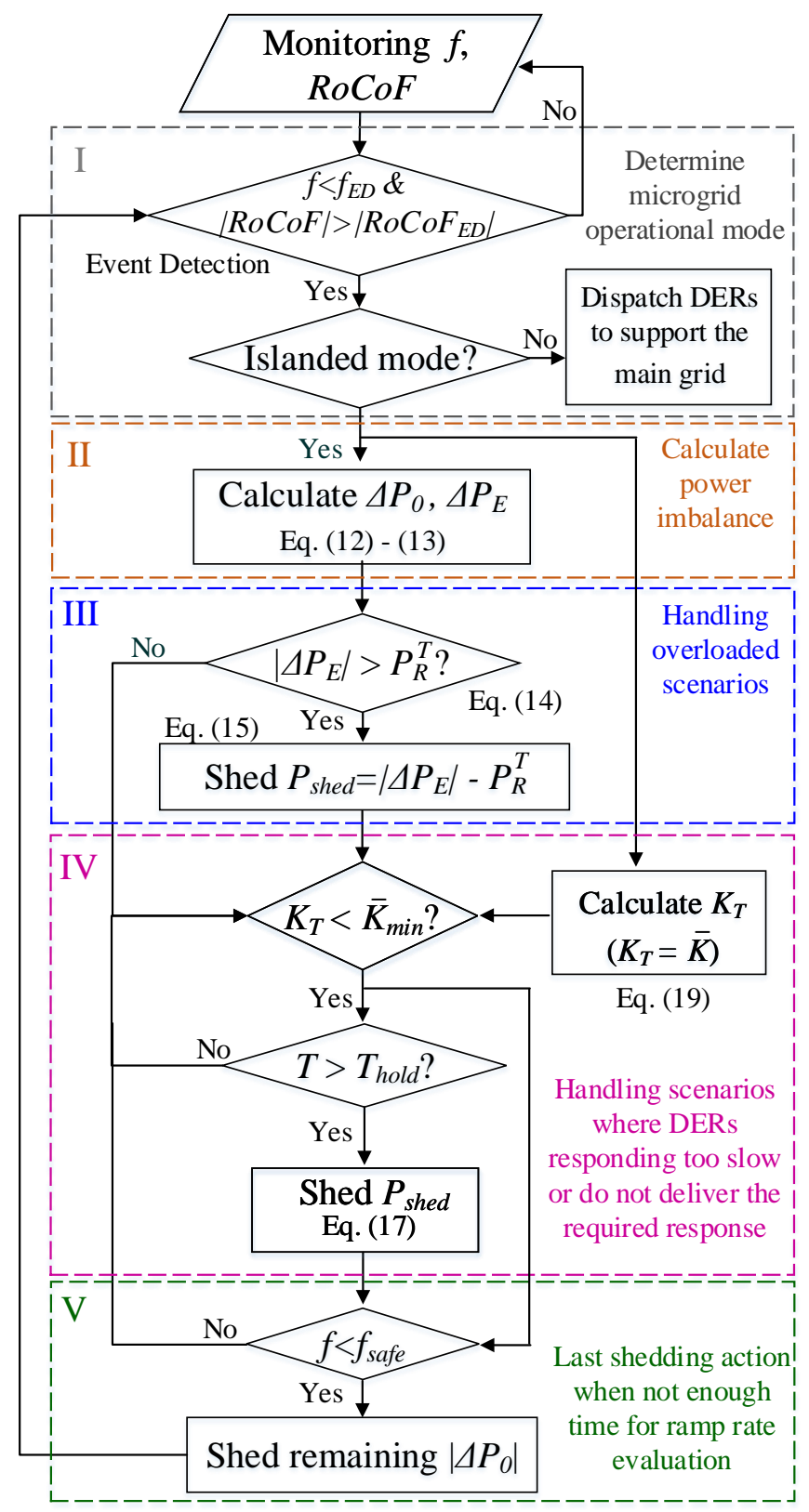

Fig. 2. Algorithm of the proposed load shedding scheme

For load shedding purpose, it is also important to estimate the event size, i.e. the largest magnitude of the power imbalance during the event, and this can be calculated using the measured maximum RoCoF, i.e. RoCoF $F_{\max }$ with (13):

$$
\left|\Delta P_{E}\right|=\frac{2 \sum H_{S M}^{i} S_{S M}^{i}}{f_{n}} \times\left|R o C o F_{\max }\right|
$$

\section{Stage III: load shedding to avoid overloading}

Assuming there are $n$ DERs in the microgrid capable of providing frequency response and the $i^{\text {th }}$ DER has a reserve active power of $P_{R}^{i}$, then the total reserve power available is:

$$
P_{R}^{T}=\sum_{i=1}^{n} P_{R}^{i}
$$

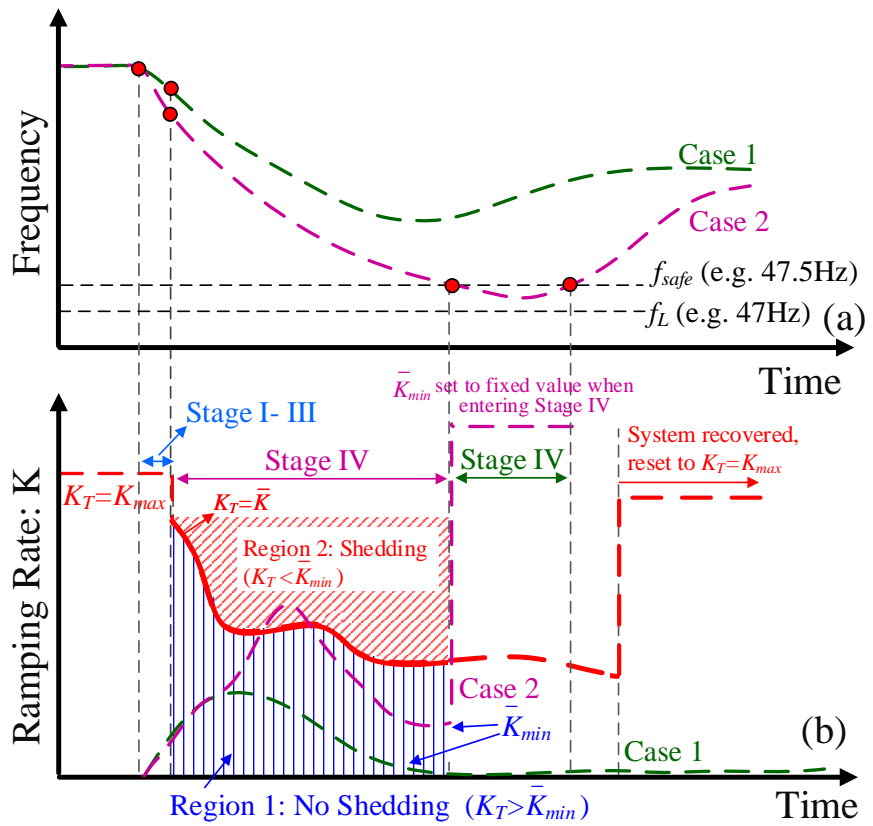

Fig. 3. Illustration of the operation of the proposed load shedding scheme

When an under-frequency event is detected, the load shedding algorithm will check if there is sufficient reserve to supply the power deficit by comparing $P_{R}^{T}$ with $\left|\Delta P_{E}\right|$ based on (15). If there is not sufficient reserve (i.e. $\left|\Delta P_{E}\right|>P_{R}^{T}$ ), then a load shedding action is required as the system will not be able to supply the total demand. The scheme will shed the load by $\left|\Delta P_{E}\right|-P_{R}^{T}$. If there is sufficient reserve (i.e. $\left|\Delta P_{E}\right| \leq P_{R}^{T}$ ), this means, from the information provided to the microgrid management system, that available resources have sufficient reserve to meet the demand. The existing load shedding schemes [3], [11]-[14] will consider that no load shedding action is required. However, from the analysis presented in Section II, it is evident that only shedding the overloaded amount may not be sufficient. In fact, even if the system is not overloaded, if DERs' active power ramping rates are too slow, the system may still fail to survive due to low frequency disconnection of DERs [40].

$$
P_{\text {shed }}= \begin{cases}0 M W & \left|\Delta P_{E}\right| \leq P_{R}^{T} \\ \left|\Delta P_{E}\right|-P_{R}^{T} & \left|\Delta P_{E}\right|>P_{R}^{T}\end{cases}
$$

\section{E. Stage IV: load shedding based on DERs' active power ramping rates}

From (11), it is known that to avoid frequency dropping to the limit $f_{L}$, a minimum average ramp rate $\bar{K}_{\text {min }}$ is required.

Assuming that the DERs have an overall ramping rate of $K_{T}$, then if $K_{T}>\bar{K}_{\text {min }}$, it means the DERs are responding at a rate that could avoid frequency dropping to $f_{L}$, so no load shedding is needed. However, when $K_{T}<\bar{K}_{\text {min }}$, shedding action will inevitably be required to bring the system back to the state where the resources would have sufficient response speed to mitigate frequency drops, so substituting $\bar{K}_{\min }$ with $K_{T}$ in (11): 


$$
K_{T}=\frac{\left(\left|\Delta P_{0}\right|-P_{\text {shed }}\right)^{2} \times f_{n}}{4 \times\left(f_{0}-f_{L}\right) \times \sum H_{S M}^{i} S_{S M}^{i}}
$$

Therefore, $P_{\text {shed }}$ can be calculated using (17):

$$
P_{\text {shed }}=\left|\Delta P_{0}\right|-\sqrt{\frac{4 \times\left(f_{0}-f_{L}\right) \times K_{T} \times \sum H_{S M}^{i} S_{S M}^{i}}{f_{n}}}
$$

The value of $K_{T}$ could be different when the load shedding scheme operates at different stages. As illustrated in Fig. 3, before and after the frequency event, the system is at steady state, so $K_{T}$ is set to be a fixed value of $K_{\max }$, which is the total maximum ramp rate that the DERs can achieve. This is to avoid unnecessary continuous calculation and setting the maximum value for $K_{T}$ also provides an extra level of security against mal-tripping.

For each DER, the maximum ramp rate $K_{\max }^{i}$ that can be achieved for a power increase of $\Delta P_{r e f}^{i}$ can be determined by applying a step change of $\Delta P_{r e f}^{i}$ in the power reference, and measure the speed of the active power response against the command. For example, for $f_{L}=47 \mathrm{~Hz}, f_{n}=50 \mathrm{~Hz}$ and $R_{i}=0.05$, using (1), $\Delta P_{\text {ref }}^{i}$ can be calculated as 1.2 pu. In practice, the DER will not allow a set point beyond 1 $\mathrm{pu}$, so to test the maximum ramping rate of the DER, a step change of power reference to the maximum power reference, i.e. $1 \mathrm{pu}$, could be applied to either the physical device or in simulation, through which the maximum ramping rate $K_{\max }^{i}$ can be determined. The total max ramp rate $K_{\max }$ of all DERs can be then be calculated as:

$$
K_{\max }=\sum_{i=1}^{n} K_{\max }^{i}
$$

As the DERs are not able to maintain their response rate at $K_{\max }$. Therefore, after the event is detected and the overloaded condition is checked in Stages I-III, the algorithm will dynamically evaluate the average ramp rate of the DERs with (19), which calculate the average rate of active power that has been injected to the system since the event detection. In Stage IV, $K_{T}$ will be set as $\bar{K}$ for load shedding actions. It should be noted that as there is time required for event detection and the DERs might have a deadband in respon there is a holding minimum time applied to change $K_{T}$ from $K_{\max }$ to $\bar{K}$. This holding time should typically be greater than the time required for event detection and the dead band delay of DERs and it is set as $0.5 \mathrm{~s}$ in this study.

$$
\bar{K}=\frac{\int_{T_{E D}}^{T_{0}}\left(\Delta P_{0}-\Delta P_{E}\right) d t}{T_{0}-T_{E D}}
$$

Fig. 3 presents two examples for illustrating the operation of the load shedding scheme. Case 1 represents a scenario where there is no shedding required, i.e. the system reserve is sufficient and the DERs' respondig rate $K_{T}$ is greater than the required $\bar{K}_{m i n}$. In case 2 , the load shedding scheme will firstly detect the event and evaluate whether there is overloading at Stage I-III. Following that, $K_{T}=\bar{K}$ is evaluated using (19) and when $K_{T}<\bar{K}_{\text {min }}$, load shedding will be triggered.

\begin{tabular}{|c|c|c|c|c|}
\hline & $S$ & $H$ & $K_{\max }^{i}$ & Droop \\
\hline CHP & $1 \mathrm{MVA}$ & $2 \mathrm{~s}$ & $0.372 \mathrm{MW} / \mathrm{s}$ & 0.05 \\
\hline Hydr & 2 MVA & $2 \mathrm{~s}$ & $0.343 \mathrm{MW} / \mathrm{s}$ & 0.05 \\
\hline BESS & $200 \mathrm{kVA}$ & N/A & $1.114 \mathrm{MW} / \mathrm{s}$ & 0.05 \\
\hline Wind & $400 \mathrm{kVA}$ & N/A & N/A & N/A \\
\hline PV & $800 \mathrm{kVA}$ & N/A & N/A & N/A \\
\hline Oth & \multicolumn{4}{|c|}{$\begin{array}{c}f_{E D}=49.8 \mathrm{~Hz}, f_{L}=47 \mathrm{~Hz}, T_{\text {hold }}=150 \mathrm{~ms} \\
R o C o F_{E D}=0.1 \mathrm{~Hz} / \mathrm{s}, f_{\text {safe }}=47.5 \mathrm{~Hz}\end{array}$} \\
\hline
\end{tabular}
When frequency approaches $f_{L}$, the calculated $\bar{K}_{\min }$ will be
TABLE I

INFORMATION ABOUT THE DERS AND LOAD SHEDDING SETTINGS

TABLE II

ACtive POWER OUtPut/CONSUMPtion BEFore Events

\begin{tabular}{ccccccc}
\hline Case & $\mathbf{1}$ & $\mathbf{2}$ & $\mathbf{3}$ & $\mathbf{4}$ & $\mathbf{5}$ & $\mathbf{6}$ \\
\hline $\begin{array}{c}\text { CHP } \\
\text { (MW) }\end{array}$ & 0.80 & 0.80 & 0.90 & 0.80 & 0.80 & 0.80 \\
\hline $\begin{array}{c}\text { Hydro } \\
\text { (MW) }\end{array}$ & 1.67 & 1.75 & 1.77 & 1.67 & 1.67 & 1.64 \\
\hline $\begin{array}{c}\text { BESS } \\
\text { (MW) }\end{array}$ & 0 & 0 & $\begin{array}{c}0 \\
\text { (Failure) }\end{array}$ & 0 & 0 & 0 \\
\hline Wind(MW) & 0.20 & 0.20 & 0.20 & 0.20 & 0.20 & 0.20 \\
\hline PV (MW) & 0.60 & 0.60 & 0.60 & 0.60 & 0.60 & 0.60 \\
\hline $\begin{array}{l}\text { Load 0 } \\
\text { (MW) }\end{array}$ & 3.40 & 3.57 & 3.50 & 3.40 & 3.40 & 3.40 \\
\hline Load 1 to 10 (each): $0.05 \mathrm{MW}$ & & & & \\
\hline
\end{tabular}

significantly increase, and based on (11), it will be an infinite value when $f_{0}=f_{L}$, so to avoid the calculation running into an infinite value, when $f_{0}$ drops to $f_{\text {safe }}, \bar{K}_{\text {min }}$ is set to a fixed value, typically much greater than $K_{T}$. The exact value is not critical as the load shedding algorithm will move to Stage $\mathrm{V}$ when frequency drops to $f_{\text {safe }}$, where $\bar{K}_{\text {min }}$ is no longer used for decision making.

\section{F. Stage V: Shedding remaining amount of power imbalance}

As mentioned previously, as load shedding actions will introduce further dynamics in the system and there could be delays in communication and measurement, so in Stage IV, a time delay $T_{\text {hold }}$ is applied after each shedding action. However, when the frequency is very close to $f_{L}$, there is not enough time for another holding period $T_{\text {hold }}$. In this case, it is desirable to shed all remaining power imbalance in the system, rather than waiting any further for the DERs to continue ramping their power. Therefore, in this scheme, a safety frequency threshold, $f_{\text {safe }}$ is used for activating Stage $\mathrm{V}$, i.e. whenever frequency drops to $f_{\text {safe }}$, the load shedding scheme will shed the remaining power imbalance without being limited by any time delay blocks. The setting of $f_{s a f e}$ is subject to the safety margin the operator wants to have, where it is set as $0.5 \mathrm{~Hz}$ above $f_{L}$ in this work.

\section{CAse Studies}

\section{A. Overview of the test configuration}

The proposed load shedding scheme has been tested using a highly realistic HiL setup as shown in Fig. 4, which contains 


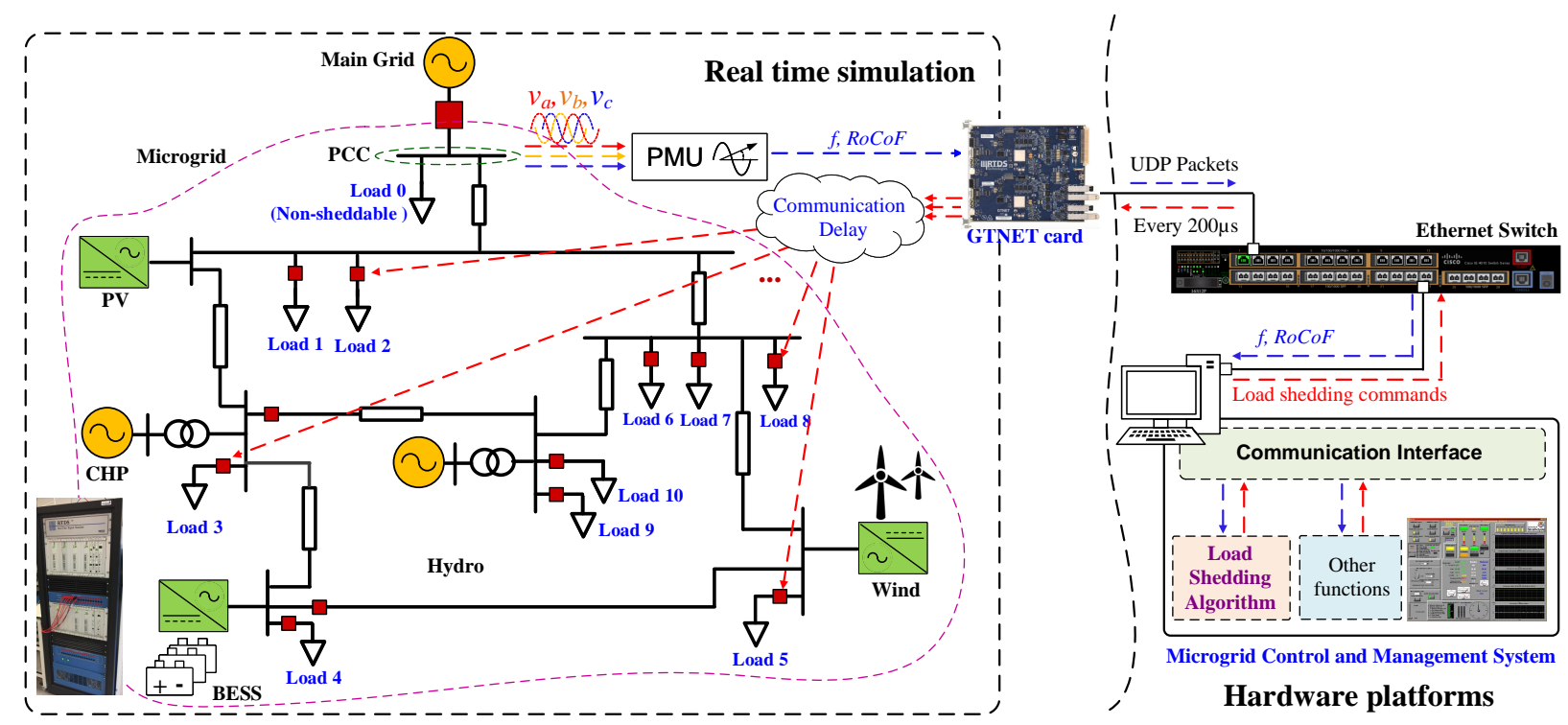

Fig. 4. Hardware-in-the-Loop test setup for validating the load shedding scheme

a real-time simulation element running in an RTDS simulator [43] and physical systems including the hardware platform executing the load shedding algorithm, along with associated communication devices. In the simulation, a modified benchmark microgrid model based on [44] is used to represent the microgrid system and a voltage source is used for emulating the main grid. In the microgrid, there are 11 loads, with one non-sheddable load and 10 sheddable loads. The DERs in the microgrid include a gas turbine-based Combined Heat and Power (CHP) unit, a mini-hydro plant, a Battery Energy Storage System (BESS), and units for representing PV and wind generation respectively. The hydro generator is modelled using a synchronous generator with the "HyGov" governor [44]; the CHP unit is also moddelled with a synchronous generator but equipped with a "GAST" governor [45] to emulate gas turbine dynamics; the BESS unit is modelled using a controllable PQ source with an additional low pass filter to emulated different levels of BESS response time (in the case study a time constant of $200 \mathrm{~ms}$ is used); for the PV and wind units, due to the intermittent nature of renewable resources and economic consideration, they are typically controlled with the maximum power tracking algorithm, thus do not normally contribute to frequency regulation, therefore they are modelled using controllable current sources to provide the specified active and reactive power. The DERs are also implemented with Under-Frequency (UF) protection with a threshold of 47 $\mathrm{Hz}$ and a delay of $0.5 \mathrm{~s}$ based on [40]. Detailed information about the DERs and the settings for the load shedding scheme are provided in Table I.

In the hardware element of the arrangement, the load shedding scheme has been developed as part of a microgrid management system as reported in [46]. For testing purposes, the load shedding algorithm is run individually on a PC as a controller, but it could also be run on a compact industrial PC platform (e.g. [47]). The simulated three-phase voltage signals at the Point of Common Coupling (PCC) are input to a Phasor
Measurement Unit (PMU) model available in RTDS [44] to measure frequency and RoCoF, which outputs measurements at 50 frames/s. In practice, the values of frequency and RoCoF could be measured with other alternative methods, and [48] presents detailed work on accurate frequency and RoCoF measurements during power system transience. The measured frequency and RoCoF were sent using the UDP protocol at a rate of every $200 \mu s$ to the controller platform, where the load shedding scheme operates with the algorithm presented in Section III. If a load shedding action is required, commands will be sent from the controller back to the RTDS to trip the required load(s) also using the UDP protocol.

For microgrid frequency control, the power imbalance at the time of islanding determines the severity of the event. Therefore, the larger of the power being imported from the main grid, the more severe the event will be. In the following case studies, various scenarios, including cases with and without sufficient reserve power, are tested to evaluate the effectiveness of the proposed load shedding scheme.

\section{B. Case 1: DERs' with sufficient reserve while the ramp up rate being too slow in containing frequency deviation}

In this test, it is demonstrated that if the DERs' active power ramping capability is not sufficiently fast during power imbalance events, it could lead to unacceptable frequency deviation. In such a scenario, even if there is enough reserve power, the microgrid will still not be able to sustain the operation and load shedding action as proposed in this paper will be required.

The system operation condition of case 1 is presented in Table II and the test results for case 1 are shown in Fig. 5. In this case, the total actual load in the system is approximately 3.98 MW. The total output from the DERs is 3.27 MW, so the power imported from the grid is approximately $0.71 \mathrm{MW}$, while the reserve power is $0.8 \mathrm{MW}$, i.e. there is sufficient reserve power from the frequency responsive units (i.e. the 

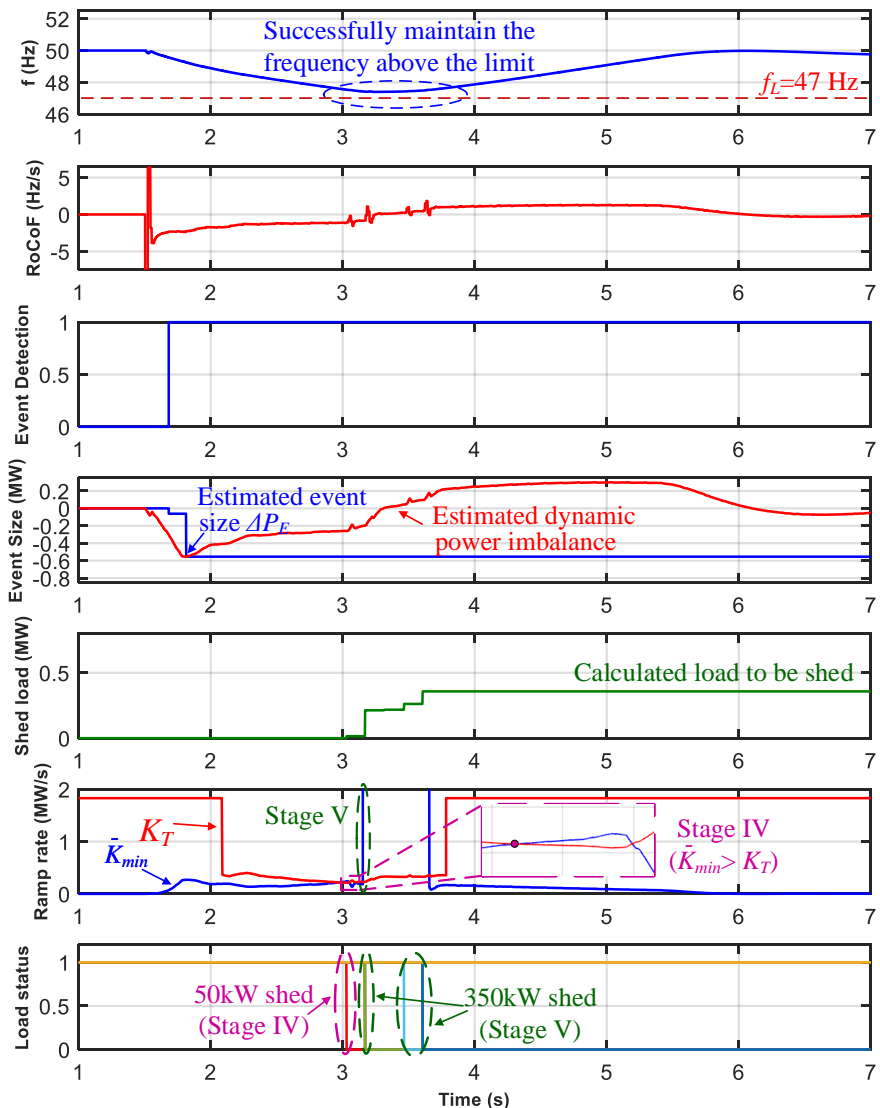

Fig. 5. Case 1 test results for validating the load shedding scheme

CHP, hydro and BESS units). At $\mathrm{t}=1.5 \mathrm{~s}$, an islanding event occurs. Conventional load shedding methods based on the comparison of the power imbalance magnitude and the reserve power will consider there is sufficient reserve, so will not trigger any shedding actions. As shown in Fig. 6, since the DERs respond very slowly to the event, it has led to the frequency dropping to below $47 \mathrm{~Hz}$. As a result, the DERs were tripped due to UF protection [40], thus leading to system collapse. With the proposed load shedding scheme as shown in Fig. 5, while there is no shedding due to the overloading condition, but at $3.03 \mathrm{~s}$, the required ramping rate $\bar{K}_{\min }$ is beyond the DERs' ramp rate $K_{T}$, which triggers a load shedding action (Stage IV as shown in Fig. 2). The calculated $P_{\text {shed }}$ as shown in the fifth plot in Fig. 5 is around $15 \mathrm{~kW}$ at this point, but as the minimum shedable load is $50 \mathrm{~kW}$, so a load of $50 \mathrm{~kW}$ is shed. Subsequently, the frequency drop to $47.5 \mathrm{~Hz}$, so the load shedding scheme moves to Stage V as presented in Fig. 2, as it considers the frequency is too close to the critical $47 \mathrm{~Hz}$ limit and it shed the remaining estimated power imbalance. As shown in Fig. 5, this leads to a disconnection of further $350 \mathrm{~kW}$. The load shedding actions successfully avoid the violation of the $47 \mathrm{~Hz}$ threshold as show in Fig. 6.

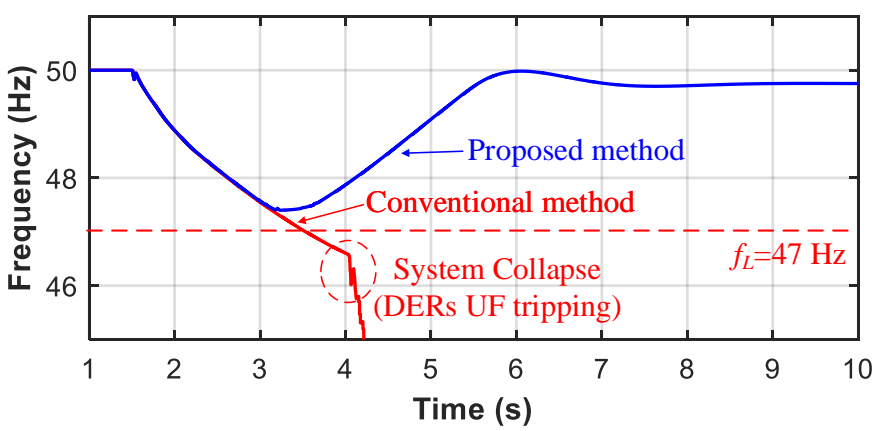

Fig. 6. Case 1: comparison of the performance between the proposed and conventional load shedding schemes
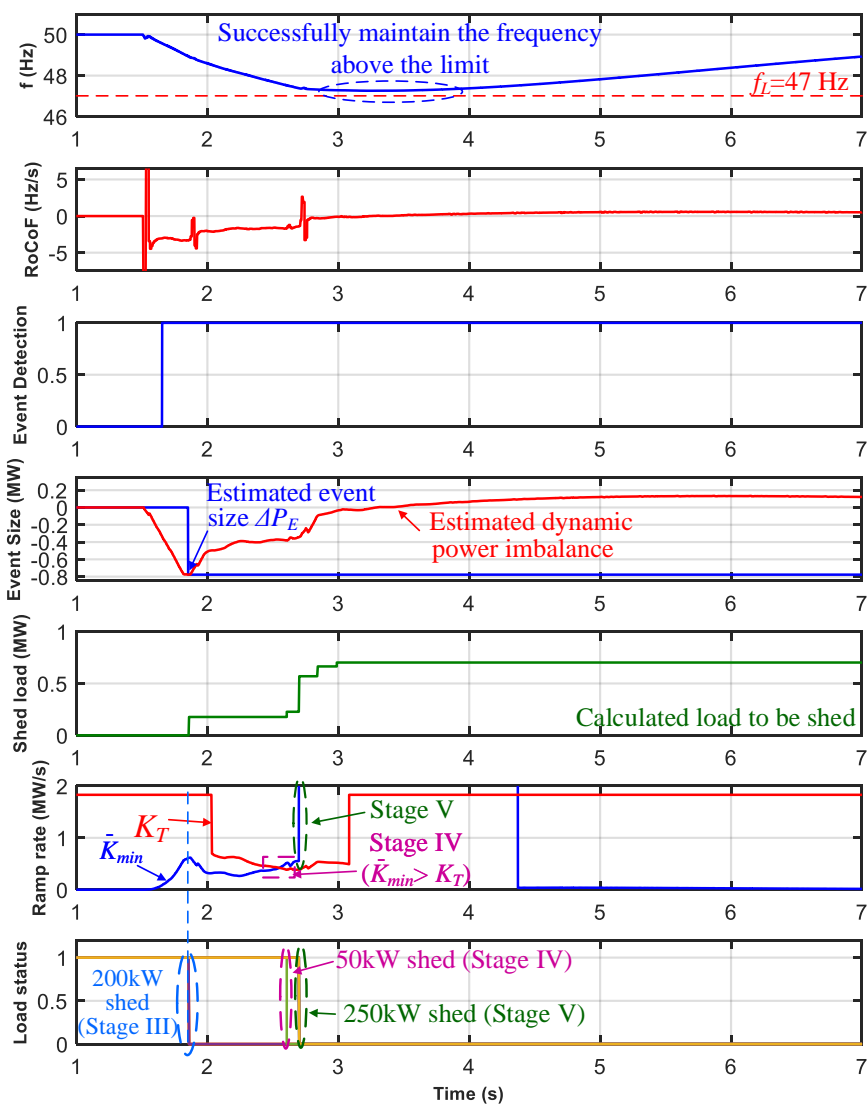

Fig. 7. Case 2: test results for validating the load shedding scheme

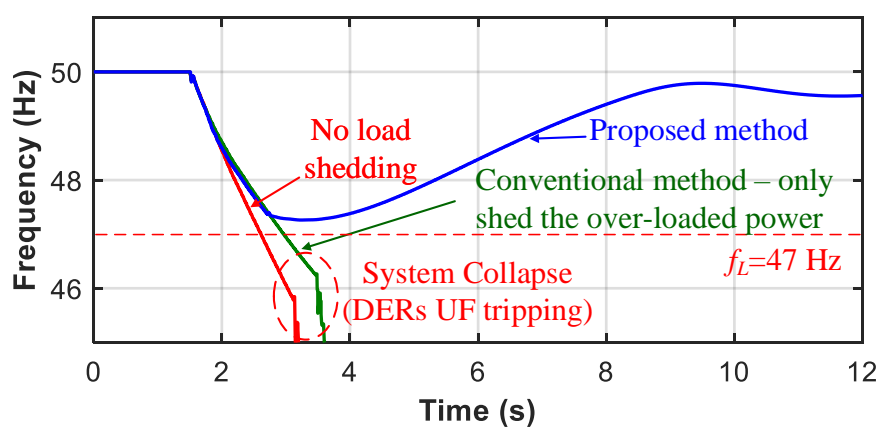

Fig. 8. Case 2: comparison of the performance between the proposed and conventional load shedding schemes 

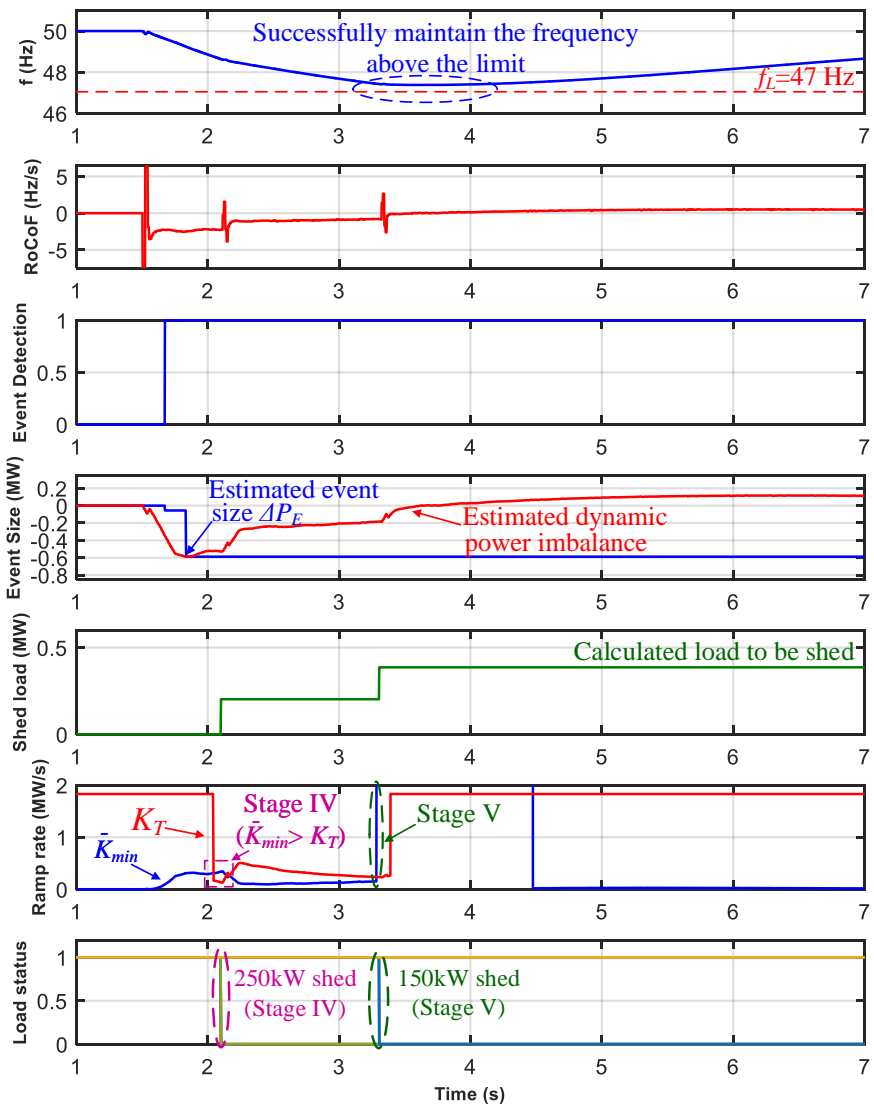

Fig. 9. Case 3: test results for validating the load shedding scheme

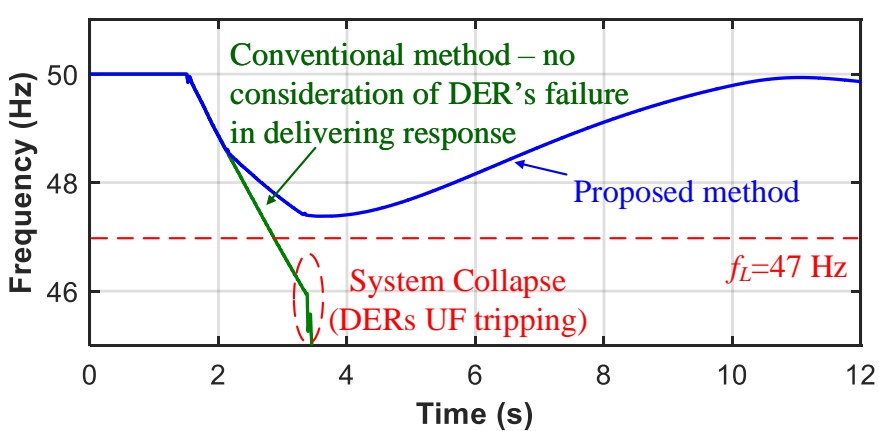

Fig. 10. Case 3: comparison of the performance between the proposed and conventional load shedding schemes

C. Case 2: both reserve power and DERs active power ramping rate are not sufficient

In Case 2, the total actual demand is approximately 4.15 MW, the DERs' output is $3.35 \mathrm{MW}$ before islanding, so the power imbalance is around 0.80 MW. In this case, the BESS system is assumed to be offline, so the total reserve power is 0.6 MW. As shown in Fig. 8, if there is no load shedding, the system will collapse due to the lack of sufficient reserve. If conventional load shedding based only on power imbalance is used, the overloaded amount is $0.2 \mathrm{MW}$, so only $0.2 \mathrm{MW}$ load is shed. It can be seen that due to the slow DERs' response, the frequency could still drop below $47 \mathrm{~Hz}$, triggering UF protection and thus a system collapse.
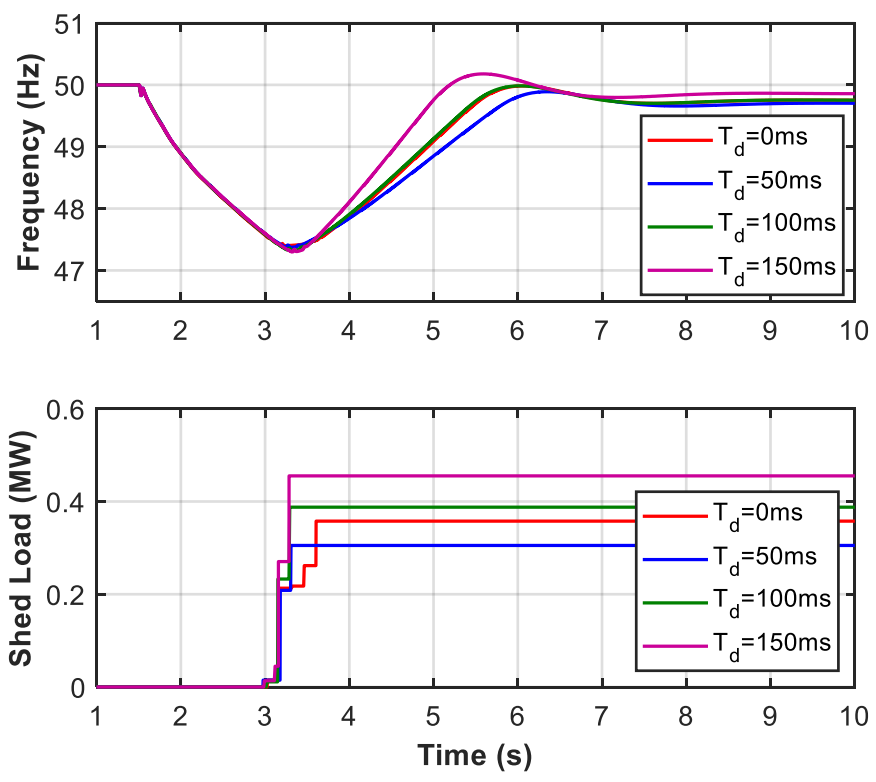

Fig. 11. Case 4: Impact of communication delays on the performance of the proposed load shedding scheme
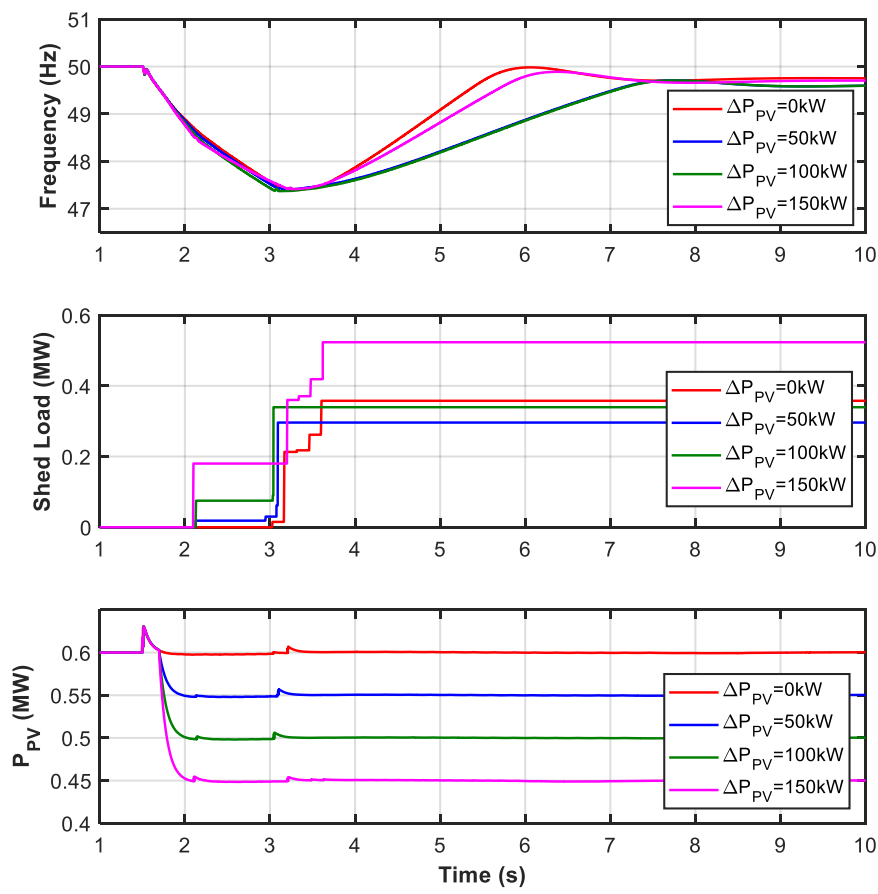

Fig. 12. Case 5: Impact of sudden change in PV active power output on the performance of the proposed load shedding scheme

With the proposed load shedding scheme, when islanding occurs, the system firstly shed 0.2 MW to avoid overloading based on Stage III as shown in Fig. 2. Subsequently, the load shedding scheme moves to Stage IV and detects that the required $\bar{K}_{\text {min }}$ is beyond the maximum active power ramping rate $K_{T}$, so it triggers a shedding of $50 \mathrm{~kW}$ at $2.61 \mathrm{~s}$. Subsequently, the frequency drops to $47.5 \mathrm{~Hz}$ and the load shedding algorithm moves to Stage $\mathrm{V}$, which triggers a shedding of a further $250 \mathrm{~kW}$ at around $2.70 \mathrm{~s}$. The load shedding actions successfully avoid the frequency dropping 
below $47 \mathrm{~Hz}$ and sustain the microgrid operation.

\section{Case 3: DERs failing in delivering required response}

In Case 3, a scenario where the BESS fails to deliver active power as expected is tested. The total demand is around 4.08 MW, the DERs' output is 3.47 MW before the islanding occurs, so the power imbalance is $0.61 \mathrm{MW}$ and the reserve power is $0.7 \mathrm{MW}$. As shown in Fig. 10, if conventional load shedding only based on power imbalance is used, it will consider that the reserve is sufficient, so no load will be shed. However, due to failure of the BESS in delivering the required power, the system will eventually collapse if no shedding is taken.

With the proposed load shedding scheme as shown in Fig. 9 , when the islanding occurs, due to the failure in the BESS active power delivery, the required ramping rate $\bar{K}_{\text {min }}$ soon exceeds the maximum ramp rate $K_{T}$, which triggers shedding of $250 \mathrm{~kW}$ of load at Stage IV. After that, the required $\bar{K}_{\text {min }}$ becomes smaller than $K_{T}$. However, at around $\mathrm{t}=3.31 \mathrm{~s}$, the frequency drops to $47.5 \mathrm{~Hz}$, which is the safety threshold as presented in Section III-F, thus shedding a further load of 150 $\mathrm{kW}$, which successfully survives the system.

\section{E. Case 4: Impact of communication delays on the perfor- mance of the proposed load shedding scheme}

As the proposed load shedding is a centralised approach, it is critical to evaluate the performance of proposed scheme with the impact of realistic communication delays. The proposed scheme only involves binary signals between the central controller and the load switches, which only requires limited bandwidth. Based on [49], low-cost communication systems have been demonstrated to be viable for microgrid protection and control purposes. According to [49], for sending a 25bit message (which is sufficient for load shedding purpose as it mainly requires binary signals) can achieve a total communication time within $50 \mathrm{~ms}$. Therefore, in this case study, the performance of the proposed load shedding scheme is evaluated with communication delays of $50 \mathrm{~ms}, 100 \mathrm{~ms}$ and $150 \mathrm{~ms}$. Case 1 is used as the base case for comparison, where there is no communication delay during load shedding. The results are shown in Fig. 11. As it can be seen from the test that, with the increase of the communication delay, while the actions of the load shedding scheme are slightly affected in terms of amounts of loads being shed and the timing of the shedding actions, the frequency of the microgrid has been successfully maintained at the required $47 \mathrm{~Hz}$ limit. The changes in the shedding actions are expected as the communication delays have led to the changed dynamic frequency behaviour during the load shedding process.

\section{F. Case 5: Impact of sudden change in PV active power output on the performance of the proposed load shedding scheme}

Due to the intermittent nature of renewable resources and economic consideration, $\mathrm{PV}$ and wind generation in microgrids are typically controlled with the maximum power tracking algorithm, thus do not normally contribute to frequency regulation. To evaluate the impact of the intermittent output from renewable generation, in this case study, case 1 is used as the base case for comparison, where there is no PV output drop during load shedding. Then, the active power output of the $600 \mathrm{kVA}$ PV unit is intentionally dropped with amounts of 50 $\mathrm{kW}(0.083 \mathrm{pu}), 100 \mathrm{~kW}(0.167 \mathrm{pu})$, and $150 \mathrm{~kW}(0.25 \mathrm{pu})$ at the time when the islanding event occurs to demonstrate the robustness of the proposed load shedding scheme. The test results are presented in Fig. 12. It can be seen from the test results that the load shedding scheme has successfully dealt with the sudden changes at PV output and maintain the frequency above the required limit $47 \mathrm{~Hz}$ critical limit.

\section{G. Case 6: Impact of different load types on the performance of the proposed load shedding scheme}

In microgrids, there could be loads with different types of characteristics connected. Two most typical types of loads are constant active and reactive power (i.e. constant PQ) and constant impedance (i.e. constant Z). For constant PQ loads, the active and reactive power are constant even with the change of voltage and frequency. This type of load is applied in the studies conducted in Case 1-5. In contrast, constant $\mathrm{Z}$ load is both dependent on frequency and voltage, i.e, the consumed power will change with the frequency and voltage variation. Therefore, when the microgrid is connected with different types of loads, the dynamic loading can vary during the frequency event, thus impacting the real time power imbalance and the frequency behaviour in the microgrid. A detailed study on the impact of loads with different voltage and frequency dependencies on load shedding actions is presented in [50].

In this study, the performance of the proposed load shedding scheme with the loads of different types is tested. Three main scenarios are investigated:1) all loads are with constant PQ type, i.e. independent of frequency and voltage changes; 2) $80 \%$ with constant PQ and 20\% with constant Z; 3) $50 \%$ with constant PQ and 50\% with constant $\mathrm{Z}$. The load and generation condition of the testing case is presented in Table II. An overview of the settings for both constant PQ and constant $\mathrm{Z}$ loads is presented in Table III. The testing results for the three scenarios are presented in Fig. 13. In all these cases, the system is with sufficient reserve to meet the power imbalance, so the conventional method purely based on power-imbalance estimation will consider the reserve is sufficient, so no load will be shed.

In the first case, where all loads are with constant PQ type, the proposed load shedding scheme successfully detects the need for load shedding and triggers shedding action to avoid frequency dropping below $47 \mathrm{~Hz}$. In the second case, $20 \%$ of the loads become constant $\mathrm{Z}$, i.e., frequency and voltage dependent, so the frequency deviation becomes slower compared with the first case. However, the proposed scheme still detects the need for shedding action and shed the load to avoid frequency dropping below $47 \mathrm{~Hz}$. In this case, the proposed scheme has adaptively reduced the amount of the load shed from $247 \mathrm{~kW}$ to $123 \mathrm{~kW}$ (the actual loads shed are $250 \mathrm{~kW}$ and $150 \mathrm{~kW}$ respectively due to the smallest load being 50 $\mathrm{kW})$. In the third case, $50 \%$ of the loads become constant $\mathrm{Z}$. As a result, the overall system loading decreased during the 

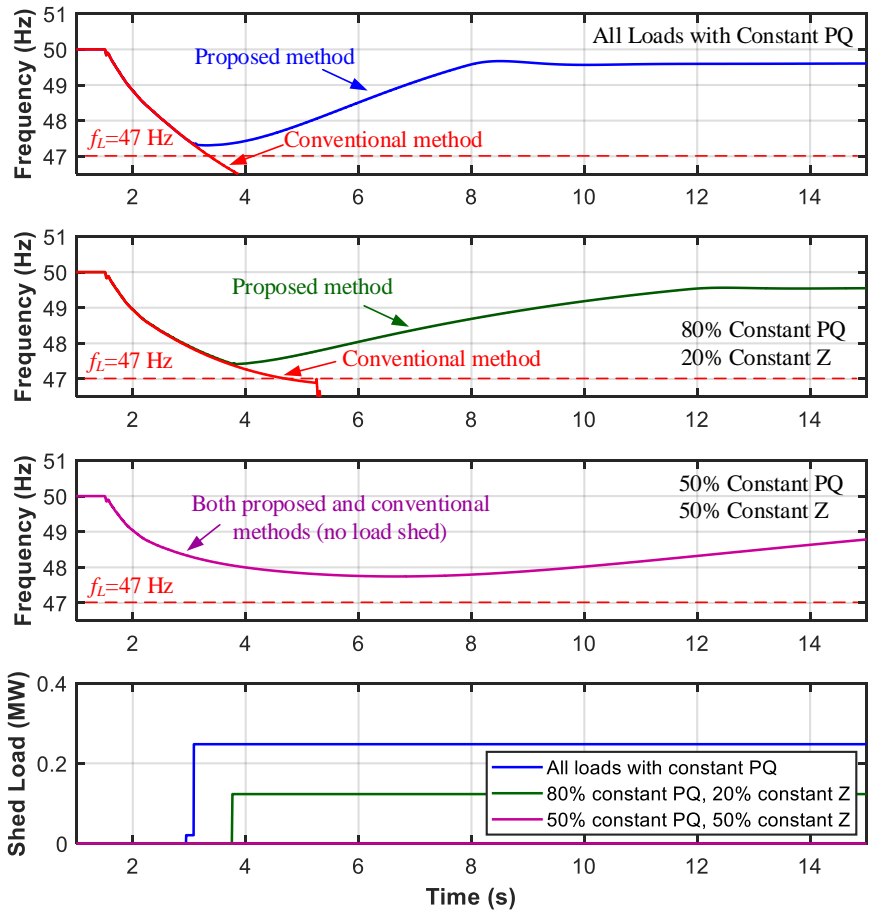

Fig. 13. Case 6: Impact of different load types on the performance of the proposed load shedding scheme

TABLE III

INFORMATION OF THE LOAD TYPES USED IN THE CASE STUDIES

\begin{tabular}{lll}
\hline Load Type & Constant PQ & Constant $\mathbf{Z}$ \\
\hline Load Connection & RL in Parallel & RL in Parallel \\
\hline P and Q output & $\begin{array}{l}\text { Frequency and volt- } \\
\text { age independent }\end{array}$ & $\begin{array}{l}\text { Dependent on both fre- } \\
\text { quency and voltage }\end{array}$ \\
\hline Nominal Voltage & $11 \mathrm{kV}$ & $11 \mathrm{kV}$ \\
\hline Base Frequency & $50 \mathrm{~Hz}$ & $50 \mathrm{~Hz}$ \\
\hline
\end{tabular}

event, thus slowing down the frequency deviation. Therefore, the system can survive without the need for shedding any loads. As it can be seen from the results, the proposed load shedding scheme has successfully made the correct decision, so no load was shed. These testing results demonstrate that the proposed scheme does present the capability in adaptively handling loads with different characteristics to ensure a certain level of security (i.e. avoiding unnecessary shedding), while maintaining a high level of dependability when shedding action is indeed required.

\section{CONCLUSiOnS}

The paper presents a new load shedding scheme that considers DERs' active power ramping capability in providing frequency responses when calculating the amount of load to be shed to maintain the frequency above the required level. From the studies conducted, a key finding is that due to the low-inertia nature of the microgrid, not only the active power reserve needs to be considered when determining the amount of the loads to be shed. If the DERs in the microgrid do not provide sufficiently fast response to the power imbalance events, the system could experience severe frequency deviation, thus total system collapse even with sufficient reserve. Therefore, the proposed load shedding scheme provides a solution by dynamically assessing the DERs' active power ramping rate to determine whether they have responded sufficiently fast to contain the frequency deviation and calculate the required amount of load needs to be shed to avoid frequency decreasing to the unacceptable level.

The presented load shedding scheme is particularly suitable for microgrids where there are a wide range of different types of DERs with diverse capability in support of frequency control. Furthermore, the proposed method is also adaptive to dynamic changes such as DERs not responding as expected, which are not considered by existing schemes. The developed load shedding scheme has been tested and validated using a realistic HiL test setup, where the results show that, with the consideration of the DER active power ramping capabilities, the load shedding action is more effective in preventing the frequency dropping to unacceptable levels due to the potential slow responses from DERs.

\section{REFERENCES}

[1] N. Bakar, M. Hassan, M. Sulaima, et al., "Microgrid and load shedding scheme during islanded mode: A review," Renewable and Sustainable Energy Reviews, vol. 71, pp. 161 - 169, 2017.

[2] J. Laghari, H. Mokhlis, M. Karimi, A. Bakar, and H. Mohamad, "An islanding detection strategy for distribution network connected with hybrid DG resources," Renewable and Sustainable Energy Reviews, vol. 45, pp. $662-676,2015$.

[3] M. Karimi, P. Wall, H. Mokhlis, and V. Terzija, "A new centralized adaptive underfrequency load shedding controller for microgrids based on a distribution state estimator," IEEE Trans. on Power Delivery, vol. 32, no. 1, pp. 370-380, Feb 2017.

[4] W. Gu, W. Liu, J. Zhu, B. Zhao, et al., "Adaptive decentralized underfrequency load shedding for islanded smart distribution networks," IEEE Trans. on Sustainable Energy, vol. 5, no. 3, pp. 886-895, July 2014.

[5] P. Anderson and M. Mirheydar, "An adaptive method for setting underfrequency load shedding relays," IEEE Trans. on Power Systems, vol. 7, no. 2, pp. 647-655, May 1992.

[6] M. Q. Ahsan, A. H. Chowdhury, S. S. Ahmed, I. H. Bhuyan, M. A. Haque, and H. Rahman, "Technique to develop auto load shedding and islanding scheme to prevent power system blackout," IEEE Trans. on Power Systems, vol. 27, no. 1, pp. 198-205, Feb 2012.

[7] P. Mahat, Z. Chen, and B. Bak-Jensen, "Underfrequency load shedding for an islanded distribution system with distributed generators," IEEE Trans. on Power Delivery, vol. 25, no. 2, pp. 911-918, April 2010.

[8] E. Dehghanpour, H. Kazemi Karegar, and R. Kheirollahi, "Under frequency load shedding in inverter based microgrids by using droop characteristic," IEEE Transactions on Power Delivery, pp. 1-1, 2020.

[9] T. Shekari, F. Aminifar, and M. Sanaye-Pasand, "An analytical adaptive load shedding scheme against severe combinational disturbances," IEEE Transactions on Power Systems, vol. 31, no. 5, pp. 4135-4143, 2016.

[10] T. Shekari, A. Gholami, F. Aminifar, and M. Sanaye-Pasand, "An adaptive wide-area load shedding scheme incorporating power system real-time limitations," IEEE Systems Journal, vol. 12, no. 1, pp. 759767, 2018.

[11] V. V. Terzija, "Adaptive underfrequency load shedding based on the magnitude of the disturbance estimation," IEEE Trans. on Power Systems, vol. 21, no. 3, pp. 1260-1266, 2006.

[12] W. Gu, W. Liu, C. Shen, and Z. Wu, "Multi-stage underfrequency load shedding for islanded microgrid with equivalent inertia constant analysis," International Journal of Electrical Power and Energy Systems, vol. 46, pp. 36 - 39, 2013.

[13] M. Karimi, H. Mohamad, H. Mokhlis, and A. Bakar, "Under-frequency load shedding scheme for islanded distribution network connected with mini hydro," Int. Journal of Electrical Power and Energy Systems, vol. 42, no. 1, pp. 127 - 138, 2012. 
[14] J. Laghari, H. Mokhlis, M. Karimi, A. Bakar, and H. Mohamad, "A new under-frequency load shedding technique based on combination of fixed and random priority of loads for smart grid applications," IEEE Trans. on Power Systems, vol. 30, no. 5, pp. 2507-2515, 2015.

[15] S. Huang and C.-C. Huang, "An adaptive load shedding method with time-based design for isolated power systems," International Journal of Electrical Power and Energy Systems, vol. 22, no. 1, pp. 51 - 58”, 2000.

[16] J. Ford, H. Bevrani, and G. Ledwich, "Adaptive load shedding and regional protection," International Journal of Electrical Power and Energy Systems, vol. 31, no. 10, pp. 611 - 618, 2009.

[17] S. Nourollah and G. Gharehpetian, "Coordinated load shedding strategy to restore voltage and frequency of microgrid to secure region," IEEE Trans. on Smart Grid, vol. 10, no. 4, pp. 4360-4368, 2019.

[18] X. Wu, S. Feng, and P. Jiang, "Distributed coordination load shedding of islanded microgrids based on sub-gradient algorithm," IEEE Access, vol. 5, pp. $27879-27886,2017$.

[19] P. He, B. Wen, and H. Wang, "Decentralized adaptive under frequency load shedding scheme based on load information," IEEE Access, vol. 7 pp. 52007-52014, 2019.

[20] M. Marzband, M. M. Moghaddam, M. F. Akorede, and G. Khomeyrani, "Adaptive load shedding scheme for frequency stability enhancement in microgrids," Electric Power Systems Research, vol. 140, pp. 78-86, 2016. [Online]. Available: https://www.sciencedirect.com/science/article/pii/S0378779616302450

[21] D. Kottick, "Neural-networks for predicting the operation of an underfrequency load shedding system," IEEE Trans. on Power Systems, vol. 11, no. 3, pp. 1350-1358, 1996.

[22] R. Hooshmand and M. Moazzami, "Optimal design of adaptive under frequency load shedding using artificial neural networks in isolated power system," International Journal of Electrical Power and Energy Systems, vol. 42, no. 1, pp. 220 - 228, 2012.

[23] Y. Hong and S. Wei, "Multiobjective underfrequency load shedding in an autonomous system using hierarchical genetic algorithms," IEEE Trans. on Power Delivery, vol. 25, no. 3, pp. 1355-1362, 2010.

[24] Y. Hong, M. Hsiao, Y. Chang, et al, "Multiscenario underfrequency load shedding in a microgrid consisting of intermittent renewables," IEEE Trans. on Power Delivery, vol. 28, no. 3, pp. 1610-1617, 2013.

[25] C. Wang, H. Yu, L. Chai, H. Liu, and B. Zhu, "Emergency load shedding strategy for microgrids based on dueling deep q-learning," IEEE Access, vol. 9, pp. 19707-19715, 2021.

[26] C. Wang, S. Mei, Q. Dong, R. Chen, and B. Zhu, "Coordinated load shedding control scheme for recovering frequency in islanded microgrids," IEEE Access, vol. 8, pp. 215 388-215 398, 2020.

[27] Q. Wang, F. Li, Y. Tang, and Y. Xu, "Integrating model-driven and data-driven methods for power system frequency stability assessment and control," IEEE Transactions on Power Systems, vol. 34, no. 6, pp. $4557-4568,2019$.

[28] X. Wu, S. Feng, and P. Jiang, "Distributed coordination load shedding of islanded microgrids based on sub-gradient algorithm," IEEE Access, vol. 5, pp. $27879-27886,2017$.

[29] Q. Huang, R. Huang, W. Hao, J. Tan, R. Fan, and Z. Huang, "Adaptive power system emergency control using deep reinforcement learning," IEEE Transactions on Smart Grid, vol. 11, no. 2, pp. 1171-1182, 2020.

[30] A. Hussain, V.-H. Bui, and H.-M. Kim, "An effort-based reward approach for allocating load shedding amount in networked microgrids using multiagent system," IEEE Transactions on Industrial Informatics, vol. 16 , no. 4 , pp. $2268-2279,2020$.

[31] V. Terzija, et al, "Direct estimation of voltage phasor, frequency and its rate of change using newton's iterative method," International Journal of Electrical Power \& Energy Systems, vol. 16, no. 6, pp. 423-428, 1994.

[32] Q. Zhou, Z. Li, Q. Wu, and M. Shahidehpour, "Two-stage load shedding for secondary control in hierarchical operation of islanded microgrids," IEEE Trans. on Smart Grid, vol. 10, no. 3, pp. 3103-3111, May 2019.

[33] A. Gholami, T. Shekari, and A. Sun, "An adaptive optimization-based load shedding scheme in microgrids," in Proceedings of the 51st Hawaii International Conference on System Sciences., 2018.

[34] P. Kundur, Power System Stability and Control. McGraw-Hill, 1993.

[35] Q. Hong, M. Nedd, S. Norris, I. Abdulhadi, M. Karimi, V. Terzija, B. Marshall, K. Bell, and C. Booth, "Fast frequency response for effective frequency control in power systems with low inertia," The Journal of Engineering, vol. 2019, no. 16, pp. 1696-1702, 2019.

[36] National Grid ESO, "Technical Report on the Events of 9 August 2019," Tech. Rep., 2019.

[37] S. Nourollah and G. B. Gharehpetian, "Coordinated load shedding strategy to restore voltage and frequency of microgrid to secure region," IEEE Transactions on Smart Grid, vol. 10, no. 4, pp. 4360-4368, 2019.
[38] S. Nourollah, F. Aminifar, and G. B. Gharehpetian, "A hierarchical regionalization-based load shedding plan to recover frequency and voltage in microgrid," IEEE Transactions on Smart Grid, vol. 10, no. 4, pp. 3818-3827, 2019.

[39] J. Choi, A. Khalsa, D. A. Klapp, M. S. Illindala, and K. Subramaniam, "Survivability of synchronous generator-based distributed energy resources for transient overload conditions in a microgrid," IEEE Transactions on Industry Applications, vol. 54, no. 6, pp. 5717-5726, 2018.

[40] Energy Network Association, "Engineering Recommendation G59: Recommendations for the Connection of Generating Plant to the Distribution Systems of Licensed Distribution Network Operators," 2011.

[41] A. Roscoe, C. Bright, S. Galloway, and G. Burt, "Increasing security of supply by the use of a local power controller during large system disturbances," in Innovative Smart Grid Technologies (ISGT Europe), 2011 2nd IEEE PES International Conference and Exhibition on, Dec 2011, pp. 1-7.

[42] A. J. Roscoe, G. M. Burt, and C. G. Bright, "Avoiding the non-detection zone of passive loss-of-mains (islanding) relays for synchronous generation by using low bandwidth control loops and controlled reactive power mismatches," IEEE Transactions on Smart Grid, vol. 5, no. 2, pp. 602$611,2014$.

[43] RTDS Technologies, "Real time digital simulation user manual," 2016.

[44] — "Microgrid modelling and simulation applications," 2016.

[45] IEEE Power System Dynamic Performance Committee , "Dynamic models for turbine-governors in power system studies," 2013.

[46] A. Roscoe, C. Bright, S. Galloway, and G. Burt, "Increasing security of supply by the use of a local power controller during large system disturbances," in IEEE PES ISGT, Dec 2011, pp. 1-7.

[47] Beckhoff Automation GmbH, "Industrial pc - for all applications," 2018.

[48] P. S. Wright, P. N. Davis, K. Johnstone, G. Rietveld, and A. J. Roscoe, "Field measurement of frequency and rocof in the presence of phase steps," IEEE Transactions on Instrumentation and Measurement, vol. 68, no. 6, pp. 1688-1695, 2019.

[49] J. Nsengiyaremye, B. C. Pal, and M. M. Begovic, "Microgrid protection using low-cost communication systems," IEEE Transactions on Power Delivery, vol. 35, no. 4, pp. 2011-2020, 2020.

[50] U. Rudez and R. Mihalic, "Analysis of underfrequency load shedding using a frequency gradient," IEEE Transactions on Power Delivery, vol. 26 , no. 2 , pp. 565-575, 2011.

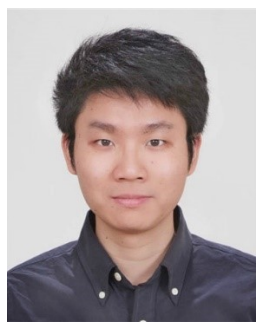

Qiteng Hong (S'11-M'15) is currently a Lecturer (Strathclyde Chancellor's Fellow) at the University of Strathclyde, Glasgow, U.K. His main research interest is on power system protection and control in future networks with high penetration of renewable generation. He received his B.Eng. (Hons) and Ph.D. degree in Electronic and Electrical Engineering in 2011 and 2015 respectively, both from the University of Strathclyde. He was the Technical Lead at the CIGRE UK Next Generation Network between 2016-2019, and is a Regular Member of the CIGRE Working Group B5.50, and IEEE Working Group P2004.

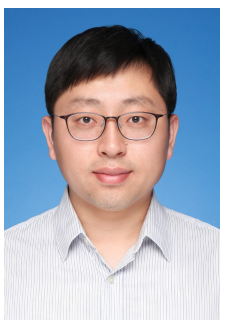

Liang Ji received his B.Eng. and Ph.D. degrees from University of Strathclyde, UK. He is presently an Associate Professor in the college of Electrical Engineering in Shanghai University of Electric Power, Shanghai. Dr Ji is the Corresponding Author of this paper. His research interests include the modelling and simulation for power system, power system protection and control. 


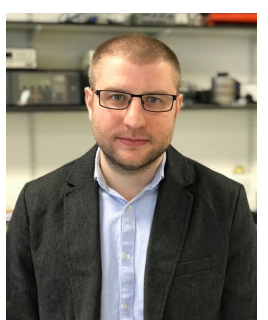

Steven M. Blair (Senior Member, IEEE) received the M.Eng. degree (Hons.) in computer and electronic systems and the Ph.D. degree in electrical engineering from the University of Strathclyde, Glasgow, U.K., in 2008 and 2013, respectively. He is currently the Head of Power Systems Technologies, Synaptec Ltd., U.K., and a Lecturer with the Department of Electronic and Electrical Engineering, Institute for Energy and Environment, University of Strathclyde. His research interests include power system protection, communications, measurements, power quality, and real-time simulation. He is also a member of IEC Technical Committee 57 Working Group 10 and the CIGRE Working Group B5.64.

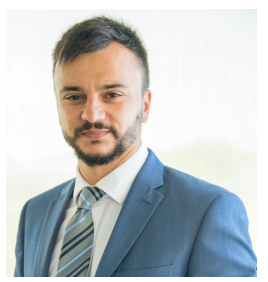

Dimitrios Tzelepis (Member, IEEE) received the BEng degree (Hons.) in electrical engineering from the Technological Education Institution of Athens, Athens, Greece, in 2013, and the MSc degree in wind energy systems and the $\mathrm{PhD}$ degree from the University of Strathclyde, Glasgow, U.K., in 2014 and 2017, respectively. He is currently a Postdoctoral Researcher with the Department of Electronic and Electrical Engineering, University of Strathclyde. His research interests include power system protection, automation and control of future electricity grids, incorporating increased penetration of renewable energy sources, and high voltage direct current interconnections. His main research methods include implementation of intelligent algorithms for protection, fault location and control applications, including the utilisation of machine learning methods and advanced and intelligent signal processing techniques. He is also interested in the application, control and protection of hybrid AC/DC grids including super-conducting feeders, non-homogeneous transmission lines, and advanced sensing technologies. Additionally, he is investigating potential solutions towards the optimised performance of active distribution networks both in off-grid and on-grid modes, to facilitate a wide suite of grid services and control capabilities.

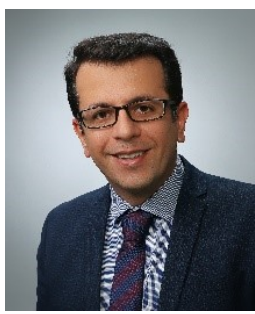

Mazaher Karimi (Member, IEEE) received his Ph.D. degree in Electrical Energy and Power System from the University of Malaya, Kuala Lumpur Malaysia, in 2013. He has been worked as a PostDoctoral research fellow at the University of Malaya in 2014 and 2015, and then joined The University of Manchester as a Research Associate, from 2016 to 2017. From 2017 to 2020, he was an Assistant Professor at Gonbad Kavous University, Iran. Currently, he is an Assistant Professor at the School of Technology and Innovations, University of Vaasa, Vaasa, Finland. His current research interests include smart grid applications; wide-area monitoring, protection, and control; distributed generation; power system stability, and frequency control.

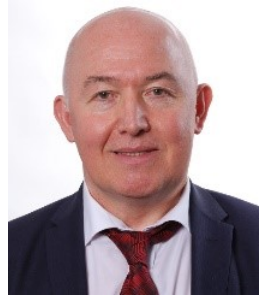

Vladimir Terzija (M'95-SM'00-F'16) was born in Donji Baraci (former Yugoslavia). He received the Dipl-Ing., M.Sc., and Ph.D. degrees in electrical engineering from the University of Belgrade, Belgrade, Serbia, in 1988, 1993, and 1997, respectively. He is a Full Professor at the Center for Energy Science and Technology, Skolkovo Institute of Science and Technology, Moscow, Russia, where he has been since 2021. From 1997 to 1999, he was an Assistant Professor at the University of Belgrade, Belgrade, Serbia. From 2000 to 2006, he was a senior specialis for switchgear and distribution automation with $\mathrm{ABB}$, Ratingen, Germany. From 2006 to 2020 he was the EPSRC Chair Professor in Power System Engineering with the School of Electrical and Electronic Engineering, The University of Manchester, Manchester, UK. His current research interests include smart grid applications; wide-area monitoring, protection, and control; multi-energy systems; substation automation; transient processes; ICT, data analytics and digital signal processing applications in power systems. Prof. Terzija is Editor in Chief of the International Journal of Electrical Power and Energy Systems, Alexander von Humboldt Fellow, as well as a DAAD and Taishan Scholar. He is the recipient of the National Friendship Award, China (2019).

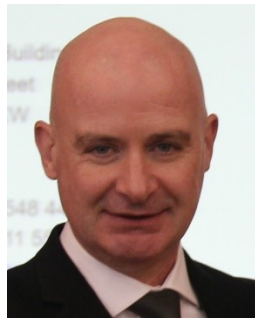

Campbell D. Booth received the B.Eng. and Ph.D. degrees in electrical and electronic engineering from the University of Strathclyde, Glasgow, U.K., in 1991 and 1996, respectively. He is currently a Professor and the Head of the Department for Electronic and Electrical Engineering, University of Strathclyde. His research interests include power system protection; plant condition monitoring and intelligent asset management; applications of intelligent system techniques to power system monitoring, protection, and control; knowledge management; and 